\title{
Spatial and temporal distribution of foot-and-mouth disease virus in the eastern zone of Tanzania
}

\author{
Authors: \\ Julius Joseph ${ }^{1}$ \\ Christopher J. Kasanga ${ }^{2}$ \\ Mmeta Yongolo ${ }^{1}$ \\ Chanasa Mpelumbe-Ngeleja \\ Raphael Sallu ${ }^{1}$ \\ Mathias Mkama ${ }^{1}$ \\ Joseph Masambu ${ }^{1}$ \\ Affiliations: \\ ${ }^{1}$ Tanzania Veterinary \\ Laboratory Agency, Tanzania \\ ${ }^{2}$ Faculty of Veterinary \\ Medicine, Sokoine University \\ of Agriculture, Tanzania \\ Correspondence to: \\ Christopher Kasanga \\ Email: \\ christopher.kasanga@sacids. \\ org \\ Postal address: \\ PO Box 3019, Chuo Kikuu, \\ Morogoro, Tanzania \\ How to cite this article: \\ Joseph, J., Kasanga, C.J., \\ Yongolo, M., Mpelumbe- \\ Ngeleja, C., Sallu, R., Mkama, \\ M. et al., 2014, 'Spatial and \\ temporal distribution of foot- \\ and-mouth disease virus in \\ the eastern zone of Tanzania', \\ Onderstepoort Journal of \\ Veterinary Research 81(2), \\ Art. \#738, 1 page. http:// \\ dx.doi.org/10.4102/ojvr. \\ v81i2.738 \\ Note: \\ Proceedings of the 2nd \\ One Health Conference in \\ Africa. Jointly organised \\ by the Southern African \\ Centre for Infectious Disease \\ Surveillance and the Tanzania \\ National Institute for Medical \\ Research, held at the Snow \\ Crest Hotel in Arusha, \\ Tanzania from 16th to 19th \\ April 2013: http://www. \\ sacids.org/kms/frontend/ \\ index.php?m=119. for Infectious Diseases \& Surveillance (SACIDS). disease (FMD) virus (FMDV) serotypes in the eastern zone of Tanzania. Observational prospective studies involving serological analysis, and FMDV antigen detection, and retrospective study on FMDV antigen detection were used in this research. Seroprevalence of antibodies to the nonstructural protein $3 \mathrm{ABC}$ of FMDV and serotype-specific antigen detection were investigated by using SVANOVIR ${ }^{\circledR}$ FMDV 3ABC-Ab ELISA and indirect-sandwich ELISA (sELISA), respectively. Serum and tissue samples were collected from cattle suspected of FMD in six districts of two regions in the eastern zone of Tanzania during the period of 2010 to 2011. A total of 41 (43.6\%) out of 94 tested sera in six district were seropositive to non-structural 3ABC protein, with the highest seroprevalence of $81.0 \%$ in Bagamoyo district, followed by Kibaha (56.2\%), Kinondoni (41.7\%), Ilala (34.8\%), Kisarawe (16.7\%) and Temeke (15.4\%) districts. Three FMDV serotypes, namely O, A and SAT 2, were detected in the eastern zone between 2001 and 2011, with type $\mathrm{O}$ being the most frequently detected serotype $(n=9 ; 60.0 \%)$ followed by type SAT $2(n=5 ; 33.3 \%)$ and type A $(n=1 ; 6.7 \%)$. These findings indicate that the eastern zone of Tanzania is predominantly infected with FMDV serotypes O, A, and SAT 2 with different spatial and temporal distribution, and that FMD outbreaks in the zone could be incriminated to at least these three serotypes. These observations imply that a rational control of FMD by vaccination in the eastern zone of Tanzania should consider incorporation of serotypes O, A and SAT 2 serotypes in the relevant vaccine(s). Further studies are required to elucidate the genetic and antigenic characteristics of circulating FMDV strains in the eastern zone of Tanzania so that an appropriate FMD control strategy can be recommended in this region.

\section{Acknowledgements}

This work was supported by the Wellcome Trust Grant WT087546MA to the Southern African Centre 